\title{
A Compact Lithium Pellet Injector for Tokamak Pedestal Studies in ASDEX Upgrade
}

\author{
R. Arredondo Parra ${ }^{1}$, R. Moreno Quicios ${ }^{1}$, B. Ploeckl ${ }^{1}$, G. Birkenmeier ${ }^{1}$, A. \\ Herrmann $^{1}$, G. Kocsis ${ }^{2}$, F. M. Laggner ${ }^{3}$, P. T. Lang ${ }^{1}$, T. Lunt ${ }^{1}$, R. Macian-Juan ${ }^{4}$, \\ V. Rohde ${ }^{1}$, G. Sellmair ${ }^{1}$, T. Szepesi ${ }^{2}$, E. Wolfrum ${ }^{1}$, W. Zeidner ${ }^{1}$, R. Neu ${ }^{1,4}$ and the \\ ASDEX Upgrade Team ${ }^{1 *}$ \\ ${ }^{1}$ Max Planck Institut für Plasmaphysik, Boltzmannstrasse 2, 85748, Garching, Germany, \\ rodrigo.arredondo@ipp.mpg.de \\ 2 Wigner RCP Institute for Particle and Nuclear Physics, 1121, Budapest, Hungary \\ 3 Institute of Applied Physics, TU Wien, Fusion@ OAW, Wiedner Hauptstr. 8-10, 1040 Vienna, Austria \\ 4 Technische Universität München, 85748, Garching, Germany
}

Experiments have been performed at ASDEX Upgrade, aiming to investigate the impact of Lithium in an all-metal-wall tokamak and attempting to enhance the pedestal operational space. For this purpose, a Lithium pellet injector has been developed, capable of injecting pellets carrying a particle content ranging from $1.82 \times 10^{19}$ atoms $(0.21 \mathrm{mg})$ to $1.64 \times 10^{20}$ atoms $(1.89 \mathrm{mg})$. The maximum repetition rate is about $2 \mathrm{~Hz}$. Free flight launch from the torus outboard side without a guiding tube was realized. In such a configuration angular dispersion and speed scatter are low, and a transfer efficiency exceeding $90 \%$ was achieved in the test bed. Pellets are accelerated in a gas gun; hence special care was taken to avoid deleterious effects by the propellant gas pulse. Therefore, the main plasma gas species was applied as propellant gas, leading to speeds ranging from $420 \mathrm{~m} / \mathrm{s}$ to $700 \mathrm{~m} / \mathrm{s}$. In order to minimize the residual amount of gas to be introduced into the plasma vessel, a large expansion volume equipped with a cryopump was added into the flight path. In view of the experiments, an optimal propellant gas pressure of 50 bar was chosen for operation, since at this pressure maximum efficiency and low propellant gas flux coincide. This lead to pellet speeds of $585 \mathrm{~m} / \mathrm{s} \pm 32 \mathrm{~m} / \mathrm{s}$. Lithium injection has been achieved at ASDEX Upgrade, showing deep pellet penetration into the plasma, though pedestal broadening has not been observed yet.

\section{INTRODUCTION}

Lithium injection performed on the DIII-D, NSTX and EAST tokamaks has yielded promising results, improving several key plasma parameters [1 - 3]: A pedestal height and width increase, and consequently an overall $\mathrm{H}$-factor increase, has been observed in DIII-D and NSTX. Additionally, plasma inventory recycling by the walls has been reduced in NSTX and EAST and ELMs have been eliminated across all reactors.

* See author list from appendix of H. Zohm, "Recent ASDEX Upgrade research in support of ITER and DEMO”, Nucl. Fusion 55(2015) 104010, doi:10.1088/0029-5515/55/10/104010 
Additionally, an optimum lithium injection rate in the $9-36 \mathrm{mg} / \mathrm{s}$ range was found in DIII-D when injecting $\mu \mathrm{g}$ sized Li granules at low speeds [3].

ASDEX Upgrade is currently the only reactor with an all-tungsten wall. Since it has been shown that lithium doping affects plasma wall interaction parameters, it is of direct interest to investigate the effect of lithium in an alltungsten-wall reactor.

The ASDEX Upgrade lithium injection experiments aimed to test the above findings on an all-metal-wall device. If successful, the results would empower ASDEX Upgrade with a new tool capable of affecting pedestal height and width, potentially enabling the testing of new experimental scenarios for a reactor. The optimum lithium injection rate determined in DIII-D served as an initial benchmark for the lithium injector at ASDEX Upgrade.

\section{DESCRIPTION OF THE INJECTOR SYSTEM}

To provide a proof of principle for characterization of lithium in an all-metal-wall tokamak, a fast track solution was developed, commissioning the injector for use with lithium pellets for only one optimized operating configuration.

The injector is capable of launching solid pellets at room temperature by a pressurized gas pulse. Pellet sizes may be of up to $2 \mathrm{~mm}$ in height and $1.5 \mathrm{~mm}$ in diameter. Compatible pellet materials are carbon, steel, molybdenum, tungsten, aluminum and lithium. Lithium pellets have been tested with the following propellant gas species: hydrogen, deuterium, helium, neon, nitrogen, oxygen, argon and krypton. When using deuterium, the injector is capable of propelling lithium pellets of up to $1.89 \mathrm{mg}$ in weight (corresponding to $1.64 \times 10^{20}$ lithium atoms) at high speeds $(420 \mathrm{~m} / \mathrm{s}$ to $700 \mathrm{~m} / \mathrm{s})$ into the plasma, with high transfer efficiencies and low speed and angular scatter when in direct free-flight launch. With the high pellet speed a deeper pellet penetration inside the plasma can be reached, allowing for a greater Li content within the pedestal region. The maximum repetition rate of the injector is $2 \mathrm{~Hz}$. A pressurized gas is used to propel the pellet, with special care being taken to ensure that the lowest achievable amount of gas enters the plasma chamber. 


\section{A. Mechanical operation}

The mechanical operation of the pellet injector hinges on its revolver-type cylinder, consisting of a revolver plate outfitted with 36 equally spaced holes of a certain specified diameter, $0.5 \mathrm{~mm}, 1 \mathrm{~mm}$ or $1.5 \mathrm{~mm}$, according to the desired pellet size (fig. 1). The plate rotates on its axis inside the injector via a check wheel operated by a push rod connected to a pneumatic piston. This mechanism ensures that the rotation of the revolver plate is $10^{\circ}$, constant with every piston upward stroke, which is calibrated by regulating the stroke length. Two openings within the injector are aligned with the revolver plate's holes. These are the pellet loading-port, used to load the holes with lithium; and the pellet chamber, into which propellant gas flows and from which the lithium pellet is propelled out of the injector.

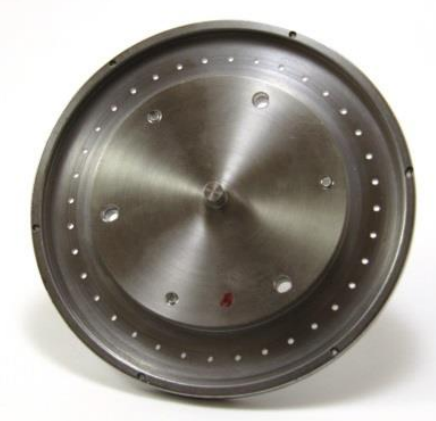

$\stackrel{1 \mathrm{~cm}}{1 \mathrm{f}}$

FIG. 1. Lithium injector revolver plate

The lithium pellet extruder is attached to the injector at the pellet loading-port, thus permitting to load the injector by extruding lithium inside the revolver plate's hole and rotating the disk from one position to the next. This extruding and rotating process is repeated until all 36 holes are filled with lithium. The pellet chamber is situated $180^{\circ}$, or 18 positions, from the pellet loading-port. Here the fast valve is attached, which releases a gas pulse, thus propelling the pellet through the barrel and out of the injector. Two arrays of light barriers detect the pellet in flight. These consist of a light-emitting diode (LED) and a photodiode. When a pellet passes through the light barrier, it is registered by the voltage drop.

Lastly, the main tube provides structural support for the injector, creating at the same time a vacuum-tight unit (fig. 2). 

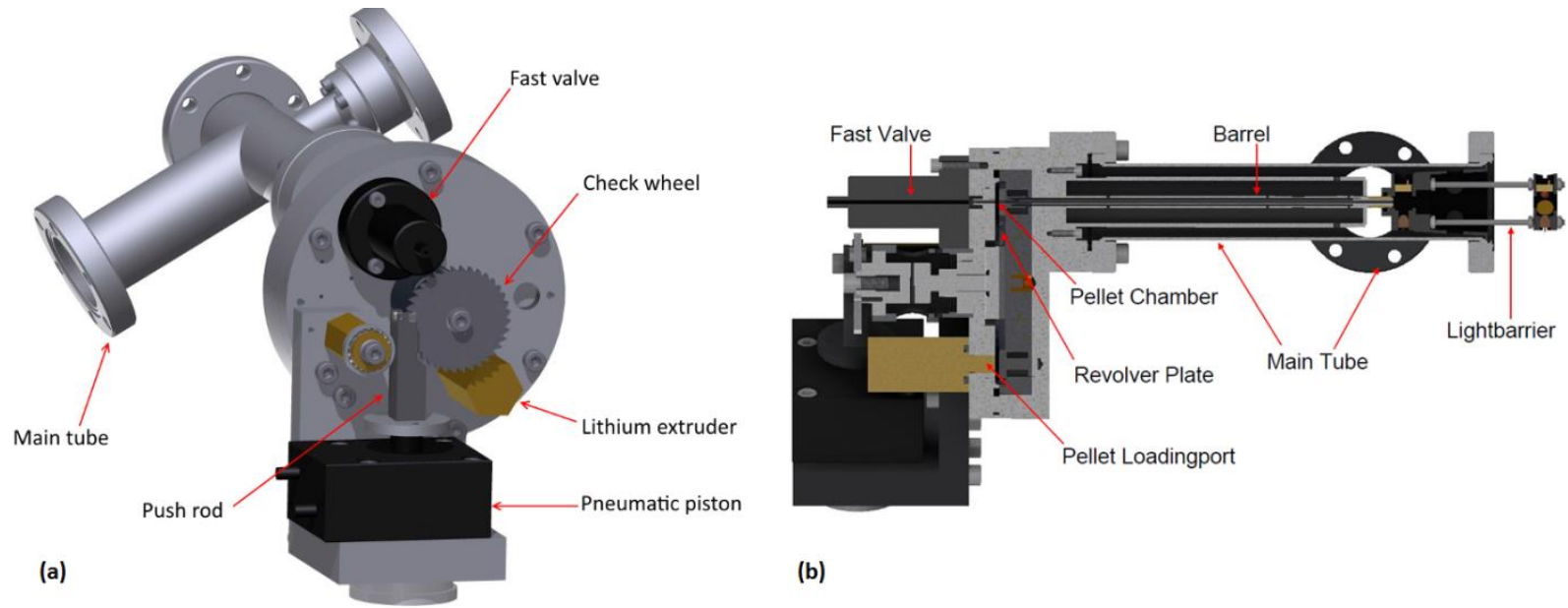

(b)

FIG. 2. 3D model of the lithium injector

\section{B. Gas injection system and pneumatics}

A pressurized bottle of gas (in our case deuterium) is used as propellant. It is connected to a pressure regulation valve, with which the desired operating pressure can be selected. A shut-off nupro valve constitutes a safety feature, ensuring that in case of malfunction, the fast valve can only "fire" a limited amount of gas: the volume between the shut-off and fast valve, approximately $2 \mathrm{~cm}^{3}$. This limited gas inventory is also of use during regular pellet shots, having the effect of reducing the propellant gas flux. The gas injection system is shown in fig. 3 .

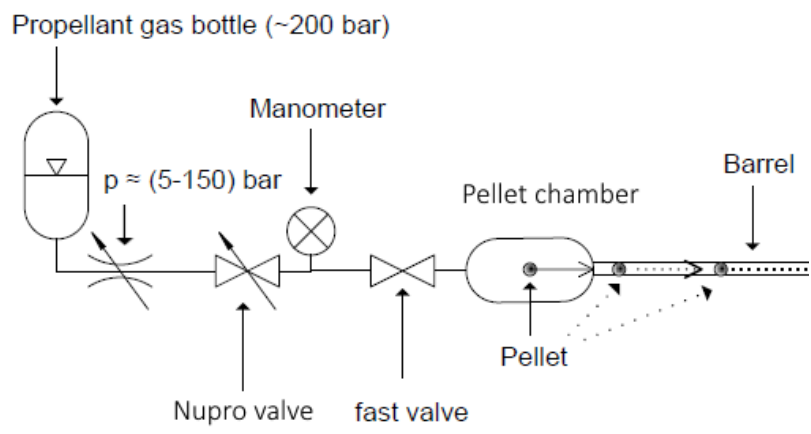

FIG. 3. Schematic of the propellant gas injection system

The electronic manometer permits remote verification of the desired propellant gas pressure before injection. The fast valve is operated by a magnetic drive reaching fully open position in $0.2 \mathrm{~ms}$ and remaining open for $2 \mathrm{~ms}$. 
The magnetic drive is controlled by a trigger unit and driven by a corresponding high current power unit. The high current power unit includes a MOSFET switched capacitor bank with a total capacitance of $10 \mathrm{mF}$ working at a nominal voltage of $115 \mathrm{~V}$. The high current power unit supplies the magnetic drive with a power of approx. $1.2 \mathrm{~kW}$ and currents of up to $14 \mathrm{~A}$, providing the required energy to actuate the magnetic drive of the fast valve.

With the given setup a pellet repetition rate of $2 \mathrm{~Hz}$ with sufficient margin is achieved, allowing for 8-10 lithium pellets per plasma discharge. The maximum designed operating pressure for this valve is 150 bar, this being the upper limit to the propellant gas pressure for the injector.

The injector pneumatic circuit is fed by a 6 bar pressure line and is operated electronically by a series of electrovalves, controlling the gate valve pertinent to the vacuum system, the shut-off valve and the switch valve that manages the lithium injector double-action piston which moves the push rod, thereby turning the revolver plate.

\section{Expansion volume}
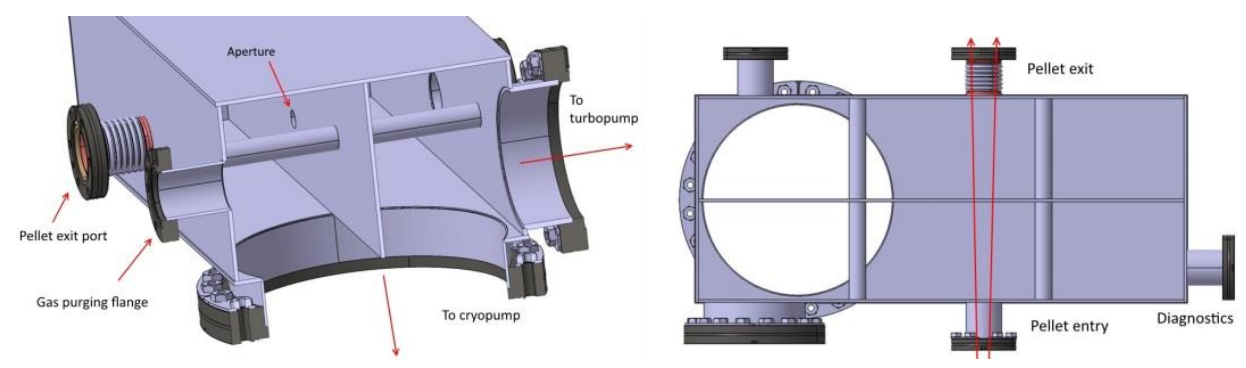

FIG. 4. Lithium injector expansion volume

It was deemed necessary in order to minimize propellant gas throughput to the torus to outfit the lithium injector with an expansion volume. Said volume (fig. 4) was modeled and calculated in accordance with the available space and geometry of the ASDEX Upgrade entry port where the injector was to be installed, providing a total expansion volume of 301 . In order to supply the necessary pumping capacity, a CTI Cryo-Torr 8 High Vacuum cryopump and a Leybold 360 turbomolecular pump were used. The latter one is connected to a Leybold Trivac D30A rough vacuum pump. The effective suction speeds for deuterium of the cryo- and turbomolecular pumps are $2500 \mathrm{l} / \mathrm{s}$ and $65 \mathrm{l} / \mathrm{s}$ respectively. The low value for the turbomolecular pump compared to its nominal suction speed $(300 \mathrm{l} / \mathrm{s})$ is due to it being connected by a $1.4 \mathrm{~m}$ tube to the expansion volume. Without the separation, the strong magnetic stray 
field near the torus could be harmful for the turbomolecular pump. The inner panel of the expansion volume divides the tank into two chambers connected only by the aperture, providing a larger pressure in the chamber connected to the lithium pellet injector during a gas pulse, which is pumped by both the cryopump and the turbomolecular pump. The second chamber, leading to ASDEX Upgrade, is pumped by the cryopump. The diameter of the aperture was adjusted to the pellet flight angular scatter, so as to minimize the amount of gas that would enter the second chamber without compromising the pellet transfer efficiency. Similarly, the width of the expansion volume was selected as the lowest possible that would permit the inclusion of a CF 200 flange. This maximizes the effective suction power of the cryopump with a minimal separation between the injector and the ASDEX Upgrade port.

Two horizontal bars were added to increase the rigidity of the expansion volume. A stress analysis was performed during the design of the vessel.

The system was partly made out of commercial vacuum components and custom made components which accommodated the specific needs of ASDEX Upgrade. During the injector characterization process in the test bed, a slightly different preliminary solution was used, comprising an expansion volume of 231 and identical vacuum pumping capacity as that projected for ASDEX Upgrade.

The system was purged with $\mathrm{N}_{2}$ to provide an inert atmosphere during lithium injector manipulation.

\section{Lithium manipulation and extrusion}

Given the reactivity of lithium with air and water, special care was taken in its manipulation, in accordance with the instructions of the Material Safety Data Sheet (MSDS).

A $\varnothing 6 \mathrm{~mm}$ lithium wire is used to load the injector. Lithium must be extruded into the revolver plate holes via the loading port. This procedure is performed manually with the use of two lithium extruders (fig. 5). These extruders work in series. The first one extrudes lithium from $\varnothing 6 \mathrm{~mm}$ to $\varnothing 2.5 \mathrm{~mm}$, while the second one extrudes lithium from $\emptyset 2.5 \mathrm{~mm}$ to the desired final diameter. This second one is directly screwed to the loading port, ensuring correct alignment with the revolver plate holes once the injector has been calibrated. 
A series of nozzles of varying diameter were made, to be fitted on the end of the $\varnothing 2.5 \mathrm{~mm}$ to $\varnothing 1.5-0.5 \mathrm{~mm}$ extruder. These allow the production of lithium rods of different diameters, necessary for the extrusion of the smaller $\varnothing 0.5 \mathrm{~mm}$ and $\varnothing 1.0 \mathrm{~mm}$ pellets. An optimized nozzle of $\emptyset 1.2 \mathrm{~mm}$ was manufactured, as it was determined that lithium expanded during loading, causing lithium buildup outside of the revolver plate holes when using the initial $\varnothing 1.5 \mathrm{~mm}$ nozzle. This buildup would eventually lead to an increased friction between the revolver plate and the lithium injector casing, allowing a progressive misalignment of the revolver plate and the pellet chamber. With the inclusion of a nozzle of smaller diameter than the pellet hole, no lithium buildup occurred (fig. 6).

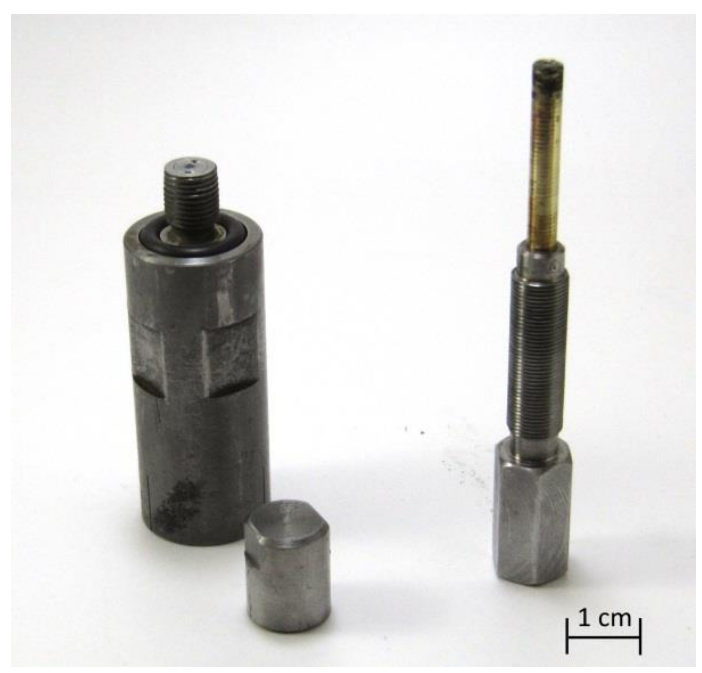

FIG. 5. Lithium extruder and extrusion nozzles

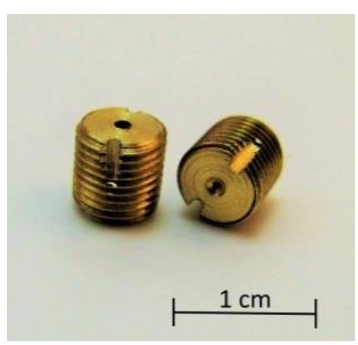

FIG. 6. Lithium extruder nozzles

\section{EXPERIMENTAL RESULTS FROM THE TEST BED}

\section{A. Pellet transfer rate tests}


Fig. 7 shows the tallied results of the pellet transfer rate tests with propellant gas pressure (pellets that exited the injector $\mathrm{v}$. total number of pellets). An uncertainty of $1 / \sqrt{n}$ was taken, where $\mathrm{n}$ represents the number of pellets in a sequence.

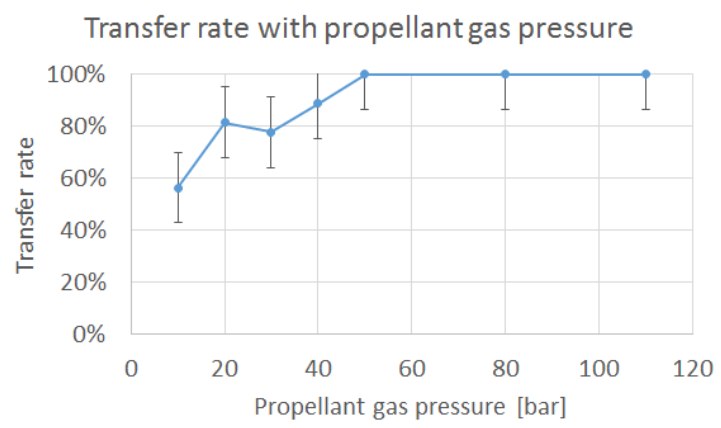

FIG. 7. Pellet transfer rates with propellant gas pressure

As shown in fig. 7, pellet transfer rates tend to increase with propellant gas pressure, plateauing at $100 \%$ approximate transfer rate for pressures of or above 50 bar. Below this value, the propellant gas may supply insufficient force on the pellet to dislodge it, as lithium pellets tend to adhere to the hole walls when in the revolver plate. The local peak at 20 bar still lies within the measurement uncertainty, as there is ample overlap between the error bars of the measurements for 20 and 30 bar.

In view of this information and with regard to the injections at ASDEX Upgrade, propellant gas pressures of or above 50 bar were recommended, as they ensure transfer rates above $90 \%$. Within this region, the choice of propellant gas pressure should be determined by other factors, as no difference in the transfer rate was observed for up to 110 bar.

\section{B. Angular scatter measurements}

The full cone scattering angle was tested for propellant gas pressures from 10 up to 110 bar, as shown in fig. 8 . The uncertainty calculations can be found in Appendix A.1, with values of approximately 5\% being obtained. 


\section{Scattering angle with propellant gas}

pressure

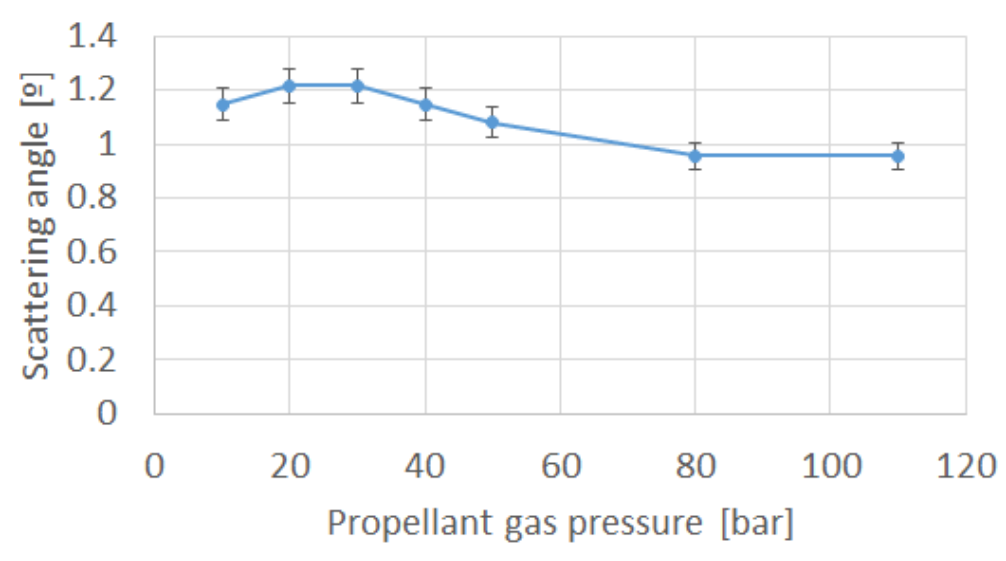

FIG. 8. Pellet scattering angle with propellant gas pressure

As can be seen in fig. 8, pellet scattering angles stay between 1.2 and $0.95^{\circ}$ for all pressures, with a trend towards lower angles at higher pressures. Due to overlap between the error bars at low pressures, the peak at 20 and 30 bar is considered non-significant. Based on this information, it is determined that propellant gas pressure has only a slight impact on pellet angular scatter, with higher propellant gas pressures being recommended for use in ASDEX Upgrade.

\section{Pellet speed and speed scatter measurements}

The average pellet speeds for each propellant gas pressure used are shown in figure 9, along with their respective speed scatter.

Pellet flight speeds range from $\sim 420 \mathrm{~m} / \mathrm{s}$ at 30 bar to $\sim 700 \mathrm{~m} / \mathrm{s}$ at 110 bar, with an average speed scatter across all propellant gas pressures of $\sigma=21 \mathrm{~m} / \mathrm{s}$. As predicted by the model (see sec. IV), higher pressures lead to higher speeds, with a sharp initial increase at low pressures and a flattening of the profile for pressures above 50 bar, since the propellant gas speed (and thus the pellet speed) cannot be greater than the sonic speed of the gas.

With this data in mind, it can be stated that high-speed (above $300 \mathrm{~m} / \mathrm{s}$ ) lithium pellets are available for use at ASDEX Upgrade, with a very low average speed scatter (at or below 5\%). While high pellet flight speeds lead to greater penetration into the plasma, pressures in the 50 - 80 bar region are recommended for general deep penetration shots, since the pellet speed increase with pressure has a very limited effect beyond this region. 


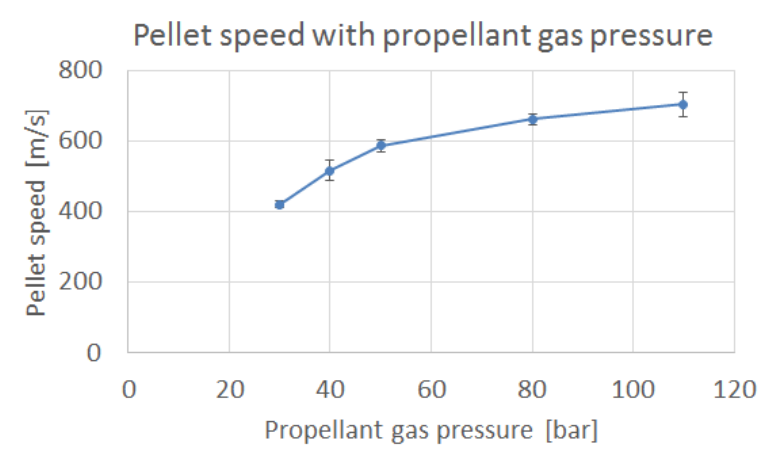

FIG. 9. Pellet flight speed and speed scatter as a function of propellant gas pressure

\section{Propellant gas flux measurements}

The peak amount of gas measured in the lithium injector expansion tank (simulating the torus) for different configurations (with or without a limiting aperture, with or without the cryopump) and pressures (10 up to 110 bar) is shown in fig. 10, as well as the reference scenario (Free expansion). It is worth noting the logarithmic scale used, as the data varied within several orders of magnitude. An overview of the test scenarios is given in table I.

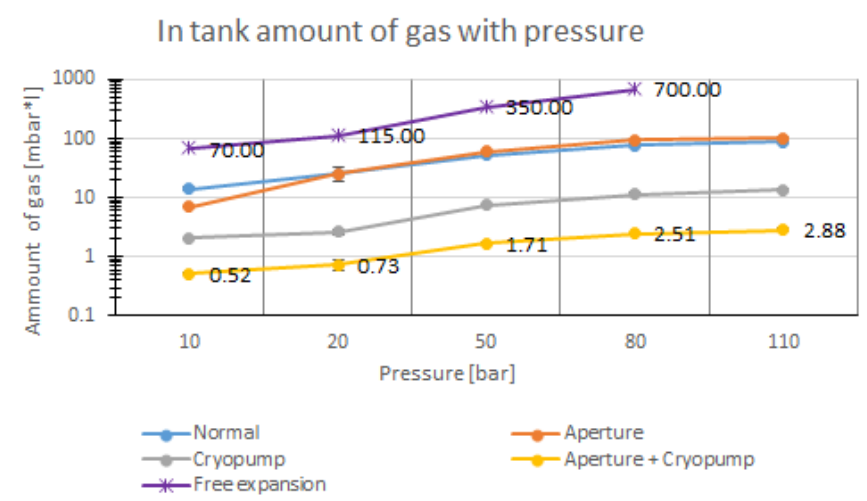

FIG. 10. Residual amount of gas in the expansion tank

TABLE I: Overview of the test scenarios for propellant gas flux measurements. Free expansion corresponds to the reference scenario

\begin{tabular}{lllll}
\hline Expansion tank & Aperture & Cryopump & Scenario & Averaged gas flux reduction factor \\
\hline no & no & no & Free expansion & 1 \\
yes & no & no & Normal & 6 \\
yes & no & yes & Cryopump & 46 \\
yes & yes & no & Aperture & 7 \\
yes & yes & yes & Aperture + Cryopump & 194 \\
\hline
\end{tabular}

For all configurations an increase in pressure is directly linked to an increase of gas content. It is also clearly visible that all configurations offer significantly better results than those with free expansion of the propellant gas. 
The lowest values are found with all systems in use (turbomolecular pump, aperture and cryopump), where a maximum reduction factor of 280 times the original values was measured for a propellant gas pressure of 80 bar: 2.51 mbar $\times 1$ versus the prior $700 \mathrm{mbar} \times 1$.

Of note is the inversion of values for the Normal and Aperture scenarios for pressures of 20 bar and beyond. The data show that in this region the use of an aperture hinders gas retention in the expansion volume, rather than facilitate it. The reason for this can be found in the operational regime of the turbomolecular pump. With the aperture in place (and without a cryopump), the expansion volume is subjected to a rough vacuum in the mbar range for a period of seconds. The turbomolecular pump's effective pumping speed is reduced to $71 / \mathrm{s}$ under these conditions, thus hampering the effective pumping speed of the expansion volume. Consequently, more gas is allowed into the vacuum tank. When the cryopump is in operation, during each gas pulse the effective suction speed of the turbomolecular pump is reduced, but the gas is almost exclusively retained in the cryopump, since in this case no lengthy low-level vacuum is achieved and the turbomolecular pump is allowed to function practically unhindered. With a cryopump, the inclusion of an aperture is beneficial, further reducing gas content compared to when it is not in place.

At expected operating pressures of between 20 and 110 bar, with an expansion volume of 30 1, turbomolecular pump, cryopump and a separating plate with an aperture, the designed system is able to provide a propellant gas flux reduction of 150 to 280 times compared to a free expansion of the gas.

\section{COMPARISON OF MEASURED PELLET VELOCITIES WITH THE ACCELERATION MODEL}

A model is used to explain the speed of propelled pellets, based on gas species and pressure used. The gas drag model yields results which fit well with the experimental measurements [4]. This model considers that solid pellets within a tube are, in the presence of a gas pulse, accelerated by the drag forces exerted by the fast moving gas as it expands. In the case of a straight tube, the maximum achievable velocity for the pellet is therefore the sound velocity of that gas, which will depend on the gas species and its temperature.

In the case of the lithium injector operation parameters employed, typical Reynolds numbers are of the order of

$10^{5}$, thus propellant gas flux can be considered a turbulent phenomenon. With this in consideration, the following equation can be used [5]: 


$$
m \frac{d v}{d t}=c_{d} A \frac{\rho_{G}(x)}{2}\left(v_{G}(x)-v(x)\right)^{2}
$$

Where $m, A$ and $v$ represent the pellet mass, cross sectional area and velocity (these last two in the direction of flight), respectively. Similarly, $c_{d}, \rho_{G}$ and $v_{G}$ are the turbulent gas drag coefficient, the gas density and the gas velocity along the flight trajectory.

By employing the transformation:

$\frac{d v}{d t}=\frac{\partial v}{\partial t}+v \frac{\partial v}{\partial x}$

And:

$\frac{\partial v}{\partial t}=0$

Equation 1 can be expressed as:

$\frac{v}{\left(v_{G}(x)-v(x)\right)^{2}} \partial v=c_{d} A \frac{\rho_{G}(x)}{2 m} \partial x$

Integrating for the whole acceleration process:

$\int_{v^{i}}^{v^{f}} \frac{v}{\left(v_{G}(x)-v(x)\right)^{2}} \partial v=\frac{c_{d} A}{2 m} \int_{0}^{L} \rho_{G}(x) \partial x$

Where $v_{i}$ and $v_{f}$ are the initial and final pellet speeds, and $L$ is the length of the barrel. With the following approximations:

$$
\begin{aligned}
& v_{G}(x)=\text { const } \\
& \rho_{G}(x)=\text { const } \\
& v^{i}=0 \\
& v^{f}=v
\end{aligned}
$$

The equation yields: 


$$
\ln \left(1-\frac{v}{v_{G}}\right)+\frac{\frac{v}{v_{G}}}{1-\frac{v}{v_{G}}}=\frac{c_{d} A \rho_{G} L}{2 m}
$$

Knowing the conditions of the gas (pressure, gas species, temperature), the geometry of the barrel and the characteristics of the pellet, the values for all the variables except $v$ can be obtained. Eq. 6 can then be solved by iterating on $v$, thus yielding the expected pellet speed according to the gas drag model. This model predicts monotonously increasing pellet speeds with propellant gas pressure, with a sharp increase for lower pressures and a more flattened increase in the case of higher pressures, asymptotically approaching the speed of sound of the propellant gas at infinite pressure.

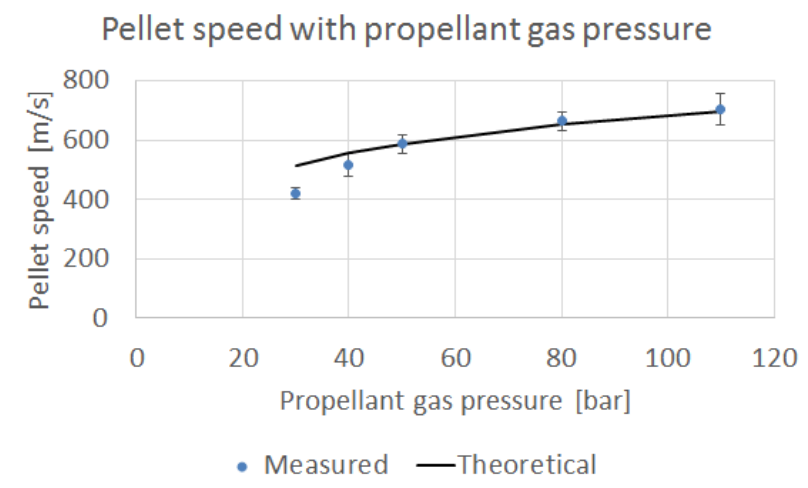

FIG. 11. Pellet speed with gas pressure, experimental results and theoretical model

The fit of the theoretical model to the experimental results is shown in fig. 11. The model correlates well with the experimental pellet speeds for propellant gas pressures of 50 bar and above. At lower pressures, pellet speeds are significantly lower than predicted. This can be attributed to pressures losses due to pellet adherence and friction. As previously mentioned, when operating at these propellant gas pressures, the force exerted on the pellets may often be insufficient to fully dislodge the pellet. Pellets that do exit the injector may therefore do so under forces lower than those predicted, leading to lower pellet speeds.

\section{APPLICATION AT ASDEX UPGRADE}

The lithium pellet injector and the specially designed vacuum system was installed in the ASDEX Upgrade sector 16 Bo port, with a direct horizontal view to the torus outboard side above its mid-plane. This port is situated horizontally on the torus outboard side, $28 \mathrm{~cm}$ above the plasma center. As already noted, said system was equipped 
with a 301 expansion volume, as well as a Cryo-Torr 8 High Vacuum cryopump and a turbomolecular pump. The setup is shown in fig. 12.

The tank position and orientation was adjusted via a laser attached to the CF 40 flange where the lithium injector would later be connected. Once the position of the tank was locked, the lithium injector is attached flush after loading to ensure consistency in the pellet flight direction.

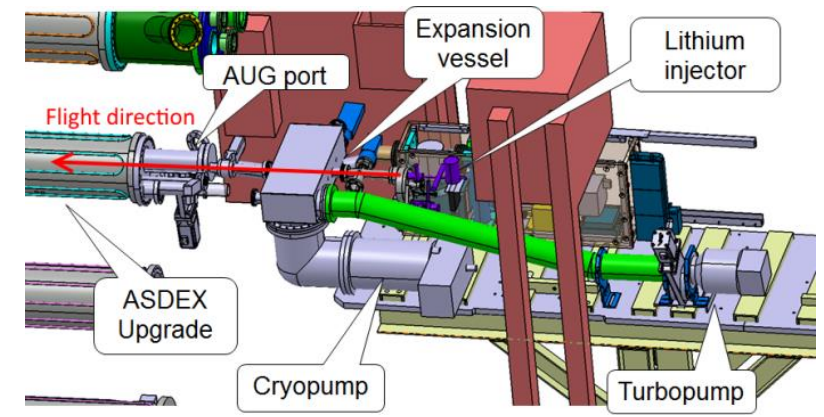

FIG. 12. Lithium pellet injector system at ASDEX Upgrade

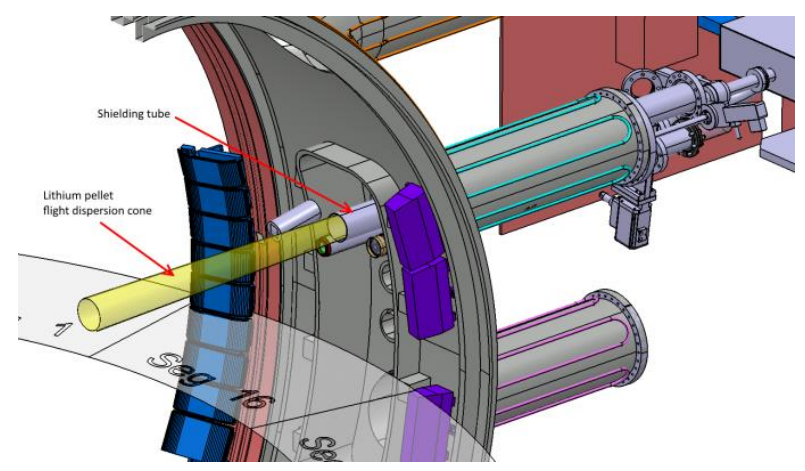

FIG. 13. Lithium pellet flight dispersion cone and shielding tube

A shielding tube was included to protect other diagnostics from possible stray pellets. Similarly to the design of aperture, the lowest possible tube diameter was chosen that could sheathe the flight dispersion cone of the lithium injector without compromising the transfer efficiency. The shielding tube and dispersion cone can be seen in figure 13. Additionally, an ablation monitor system was installed, consisting of a wide angle waveguide which captures the light coming from the torus near the sector 16 Bo port during ablation. Several cameras were fitted to provide different views of the pellet ablation in the plasma. Fig. 14 shows a horizontal view from the torus outboard side of a pellet ablation above the midplane. The ablation plume following the magnetic field line is clearly visible, with the 
characteristic green light of the lithium emission line. A camera was also fitted to provide a birds-eye view of the pellet ablation in the plasma.

With this configuration, tests were performed to characterize the injector during normal ASDEX Upgrade operation, including gas throughput into the torus and pellet transfer efficiency.

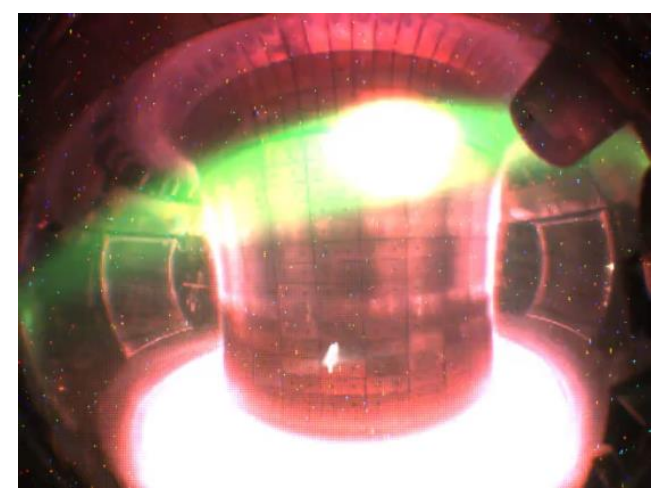

FIG. 14. Shot \#32052 at $5.572 \mathrm{~s}$ : lithium pellet injection at ASDEX Upgrade during full power phase with $\mathrm{N}_{2}$ seeding

\section{A. Gas throughput}

Propellant gas throughput to the torus was measured by firing a sequence of gas pulses without lithium pellets at an empty torus under different configurations. Propellant gas throughput was measured with and without the lithium pellet injector cryopump in use, and shooting into a pumped or unpumped torus. A limit of $10^{21} \mathrm{D}$ atoms per second was set as a benchmark goal, approximately equivalent to a weak gas puff level considered sufficiently low so as to not alter plasma behavior. Three tests were performed, shown in fig. 15.

Shot \#31790 consisted of a sequence of 11 gas pulses at $2 \mathrm{~Hz}$ while using the on-board cryopump and with the torus pumping system active. This is the intended operating regime, and is well below the propellant gas flux set as a benchmark. Shot \#31793 consisted also of a sequence of 11 gas pulses at $2 \mathrm{~Hz}$, this time with the on-board cryopump switched off, albeit maintaining a constantly pumped torus. In this configuration, propellant gas flux during the first pulses was greater than the benchmark goal, although the torus pressure plateaued soon after. Shot \#31796 consisted of a sequence of 10 gas pulses at $2 \mathrm{~Hz}$, with the on-board cryopump on, in an unpumped torus. Even in this configuration the final propellant gas throughput is of approximately $6 \times 10^{20} \mathrm{D} / \mathrm{s}$, lower than the imposed limit of $10^{21} \mathrm{D} / \mathrm{s}$. 
It can be therefore concluded that propellant gas flux to the torus has been significantly diminished to a value which should not have a detrimental impact on the physics investigations.

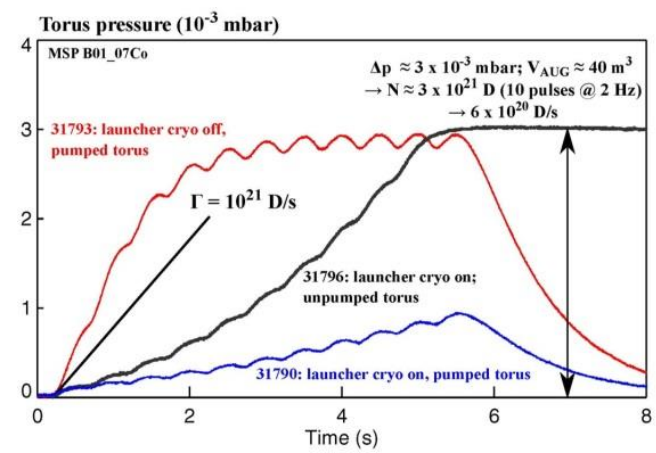

FIG. 15. Propellant gas throughput to the ASDEX Upgrade torus

\section{B. Pellet transfer efficiency and injection control}

Pellet transfer efficiency was monitored during operation at ASDEX Upgrade. This was done by registering the lithium ablation monitor signals (fig. 16, labeled Pellet monitor), and by effectuating a recount of the pellet inventory in the revolver plate after operation at ASDEX Upgrade. The final tests showed transfer rates consistent with the results obtained in the test bed, with a transfer efficiency of $\sim 94 \%$. However, not all pellets produced the same effect on the plasma, with some pellet launches showing multiple small bodies reaching the plasma. It is believed that a number of pellets may fracture during flight, not being able to penetrate into the plasma.

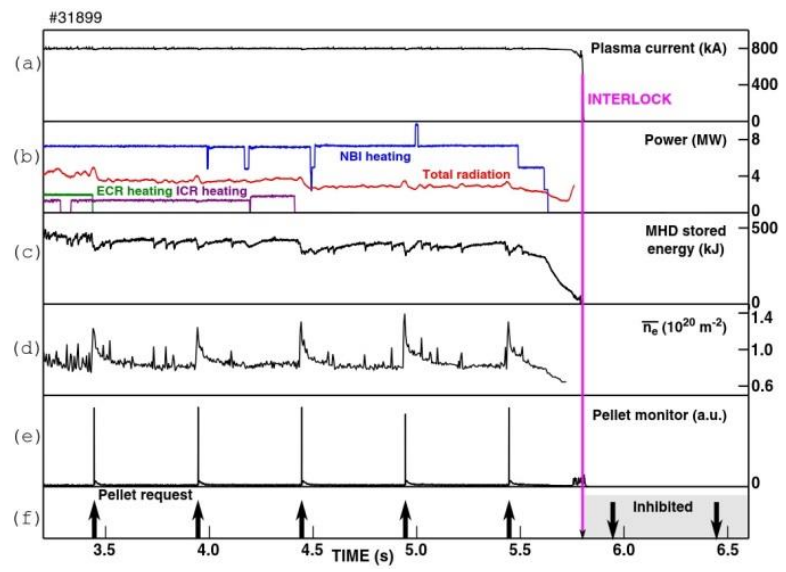

FIG. 16. Plot of signals during shot \#31899. From top to bottom: (a) plasma electric current, (b) heating power input and output, (c) stored energy in the plasma, (d) electron density, (e) lithium pellet ablation monitor signal and (f) the pellet request trigger 
Fig. 16 shows a typical plasma discharge with the injection of lithium pellets. The onset of the pellets can be seen in the lithium ablation monitor signal (e) (Pellet monitor), showing 5 sound pellets reaching the plasma.

A sound pellet can be seen to produce an electron density increase of approximately $3 \times 10^{20}$ electrons, as evidenced in the electron density plot $(\mathrm{d})\left(\overline{n_{e}}\right)$. Given the three electrons per lithium atom, this yields a lithium content of $10^{20}$ lithium atoms per pellet, consistent with the content of one full-sized lithium pellet. Additionally, the injection of a pellet into the plasma coincides with a transient dip in the stored energy in the plasma (c) (MHD stored energy), indicating that the plasma is temporarily cooled by the non-ionized pellet. The Power plot (b) shows the heating power provided to the plasma (ECRH, ICRH, NBI) and the radiated power of the plasma. Plasma current (a) describes the electric current of the plasma during the discharge.

Firing sequences are programmed prior to a plasma discharge and take place automatically once the necessary safety and operating conditions are met. If the safety interlock signal is lost, the firing sequence is interrupted and the subsequent pellets are inhibited. This can be seen in fig.16, where a loss of the interlock signal, due to a premature plasma termination, leads to the inhibition of the two remaining pellets in the sequence (f).

Instances of fractured pellets tended to increase with the number of pellets being launched. Since all remaining pellets come in contact with deuterium during each pellet launch, it is postulated that pellets may over time form a layer of lithium deuteride, which could promote cracking when launched.

\section{Impact of lithium in ASDEX Upgrade}

The effect of lithium in the plasma at ASDEX Upgrade has been observed on several diagnostics. As already mentioned, a steep and short-lived electron density increase has been noted when injecting sound pellets (fig. 16).

Pellet injection also coincides with a strong increase in the signals of the filter-photomultiplier detectors of the lithium beam emission diagnostic at the edge of the plasma, which usually measures the light emission from a Li beam via several beam lines at different observation depths, ranging from the plasma edge to $18 \mathrm{~cm}$ into the plasma. A description of the diagnostic and the lines of sight used can be found in $[6,7]$. The signal can be seen to saturate after inclusion of a pellet for all lines of sight (fig. 17). Past this strong initial increase, several short lived peaks are observed. These are in appearance similar to the small ELMs observed during regimes where magnetic perturbation 
is used [8]. However, this increase of lithium content is short lived, below the $0.5 \mathrm{~s}$ between pellets. Thus, no large lithium accumulation effects are observed during the experiments.

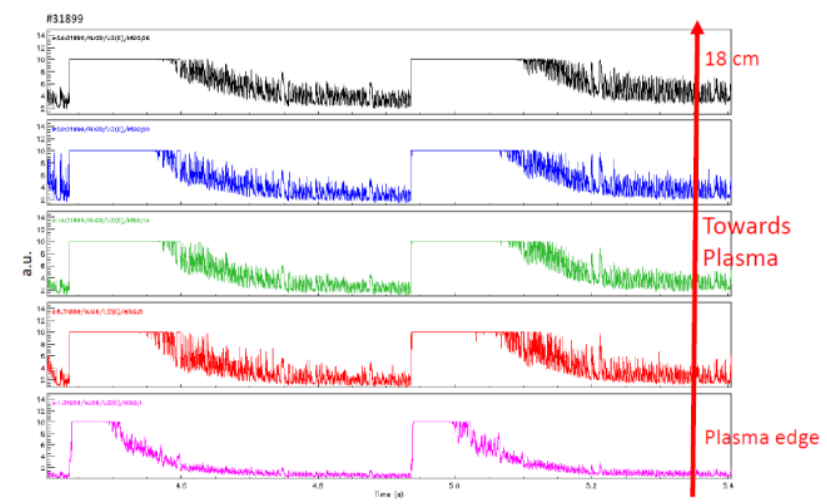

FIG. 17. Lithium line emission from the plasma edge (bottom) up to $18 \mathrm{~cm}$ inside the separatrix (top) detected with filterphotomultipliers

Lithium build-up was seen, however, between plasma discharges. Fig. 18 illustrates lithium light emission during discharges before, during and after lithium pellet injection. During each shot, the active lithium beam signal is toggled on (the Li beam signal and background Li content are seen) and off (only the background is seen). Shots \#31895 and \#31898 represent the active and background signal from the Li beam diagnostic before the injection of lithium. The peaks observed correspond to ELMs. Shots \#31899 and 31900 had lithium pellet injection, during which the background Li signal is greatly increased. Particularly, during shot \#31900 the background Li signal is so strong that the effect of the active Li beam signal cannot be observed. Shots \# 31902 and 31904 are again without Li injection. Though no lithium pellets are injected, the background intensity is still higher than before injection of lithium. Finally, shot \#31910 shows lithium intensity with a freshly boronized wall being back to the level before $\mathrm{Li}$ injection. 

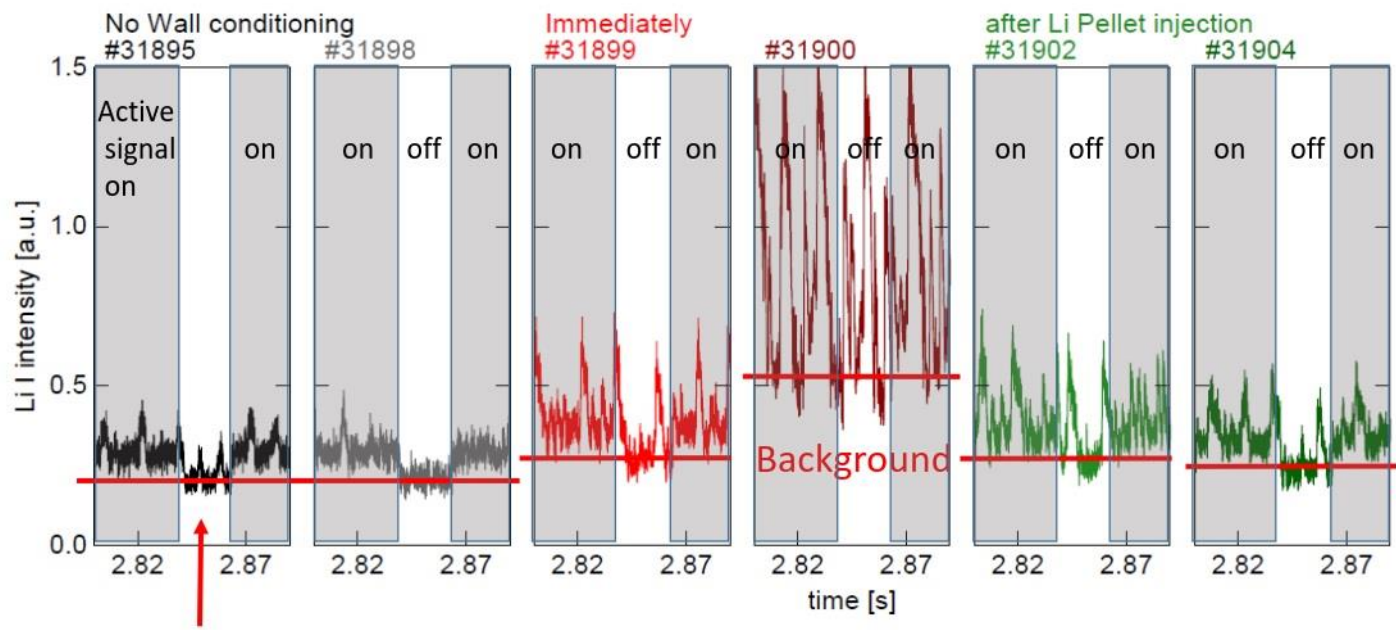

Boronized Wall

Active signal off

FIG. 18. Lithium buildup in in the signal of the Li beam emission before, during and after pellet injection

The observed lithium buildup indicates a limited wall conditioning effect, analogous to boronization, with lithium depositing on the torus walls, forming a thin layer. This effect can be seen during plasma current ramp-up in figure 19, which shows the current ramp-up in multiple discharges before and after lithium injection. Ramp-up before $\mathrm{Li}$ injection can be seen in shots \#31895 and \#31898. Ramp-up immediately after lithium inclusion is shown in shots \#31899 and \#31900, and shot \#31902 and \#31904 and occur subsequently after that. For comparison Shot \#31910 shows plasma current ramp-up in a freshly boronized torus.

With the injection of $\mathrm{Li}$, the plasma current rise is significantly faster. This effect tapers off after a few discharges. In all cases, the effect is not as pronounced as after a boronization (see\#31910).

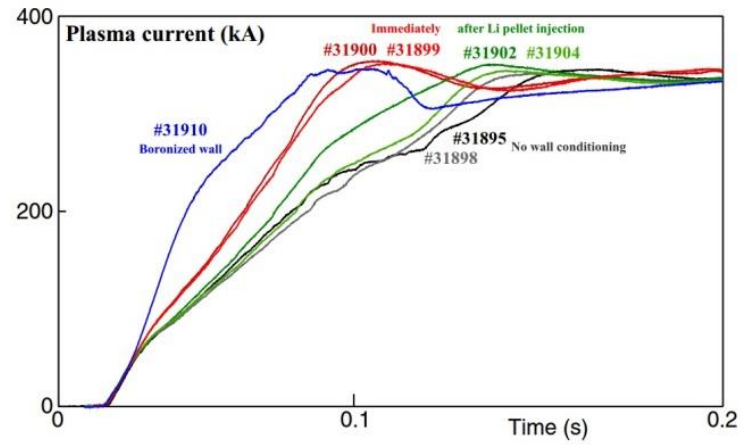

FIG. 19. Plasma current ramp-up before (\#31895 and \#31898) and after (\#31900 - \#31904) Li injection. For comparison \#31910 is shown, which was performed shortly after a boronisation 
An effect of lithium on the pedestal and on ELMs could not be observed yet, and more detailed findings regarding the physical effect of lithium pellets on ASDEX Upgrade plasmas will be published in a subsequent paper.

\section{SUMMARY}

A lithium pellet injector has been successfully characterized and tested at ASDEX Upgrade. It is capable of launching Li pellets of up to $1.89 \mathrm{mg}\left(1.64 \times 10^{20} \mathrm{Li}\right.$ atoms) at speeds between $420 \mathrm{~m} / \mathrm{s}$ and $700 \mathrm{~m} / \mathrm{s}$ into the plasma, with high transfer efficiencies and low speed and angular scatter when in direct free-flight launch. Pellets are launched via a gas pulse from a pressurized bottle. Deuterium was used as propellant gas at ASDEX Upgrade, since this is the main plasma species. The injector has a maximum repetition rate of $2 \mathrm{~Hz}$. The magazine has capacity for 36 pellets, and is loaded manually. Gas throughput to the torus has been reduced to acceptable values for the plasma via the inclusion of a custom-built expansion volume together with a turbomolecular pump and cryopump. Lithium pellet ablation has been observed inside the plasma, though a fraction of pellets tended to break before penetrating into the plasma. While no lithium accumulation was observed between pellets, lithium did accumulate in the device during plasma discharges. A wall conditioning effect has been observed, akin to boronization. The effect of lithium pellets on the pedestal and on ELMs could not be observed at this time.

\section{ACKNOWLEDGMENTS}

The team at ASDEX Upgrade would like to thank Andreas Seiler, Andreas Wöls, Bertram Brücker, Andreas Bolland, Manfred Sochor, Wolfgang Weisbart, Michael Beck and Leonhard Leppin for their help and support in this project. F. M. Laggner is a fellow of the Friedrich Schiedel Foundation for Energy Technology.

The work leading to this article was funded by the European Atomic Energy Community and is subject to the provisions of the European Fusion Development Agreement

\section{APPENDIX A: CALCULATION OF ANGULAR SCATTER UNCERTAINTY}

Pellet flight angular scatter is determined through the impact position left on the catching box plate. It is calculated from the scatter diameter on the plate $d$ and the distance from the lithium injector pellet chamber to the

plate $\mathrm{l}=303 \mathrm{~mm}$ through equation $\alpha=2 \times \arctan \left(\frac{d / 2}{l}\right)$. The measurement uncertainty in this test is thereby dependent 
on both of these values, as well as their respective measurement uncertainties. These are taken to be the minimum measuring unit of the instrument (in this case $1 \mathrm{~mm}$, using a standard ruler) or $5 \%$ of the total measurement, whichever may prove greatest of the two, thus providing a conservative approximation.

$$
\Delta \alpha=\left|\frac{\partial \alpha}{\partial d}\right| \times \Delta d+\left|\frac{\partial \alpha}{\partial l}\right| \times \Delta l=\left|\frac{l}{d^{2}+l^{2}}\right| \times \Delta d+\left|-\frac{d}{d^{2}+l^{2}}\right| \times \Delta l
$$

Where:

$$
\begin{aligned}
& l=0.303 \mathrm{~m} \\
& \Delta l=0.05 \times l=0.015 \mathrm{~m} \\
& \Delta d=10^{-3} \mathrm{~m}
\end{aligned}
$$

Table II shows the uncertainty in the angular scatter measurement.

\begin{tabular}{llll}
\multicolumn{2}{l}{ TABLE II: Angular scatter uncertainty } & \\
\hline Angle $\left[^{\circ}\right]$ & $\mathrm{d}[\mathrm{m}]$ & Error $\left[^{\circ}\right]$ & Relative error $(\%)$ \\
\hline 0.9 & 0.0048 & 0.048 & 5.32 \\
0.95 & 0.0050 & 0.050 & 5.30 \\
1 & 0.0053 & 0.053 & 5.28 \\
1.05 & 0.0056 & 0.055 & 5.26 \\
1.1 & 0.0058 & 0.058 & 5.25 \\
1.15 & 0.0061 & 0.060 & 5.23 \\
1.2 & 0.0063 & 0.062 & 5.22 \\
\hline
\end{tabular}

\section{APPENDIX B: CALCULATION OF PELLET SPEED UNCERTAINTY}

The pellet flight speed is measured through a set of two light barrier arrays. These have a separation of $\mathrm{s}=72$ $\mathrm{mm}$. These light barriers produce an electrical signal which is recorded on an oscilloscope with negligible delay and a time division of of $\Delta \mathrm{t}=1 \mu \mathrm{s}$. The uncertainty in the pellet speed will therefore be dependent of the time it takes the pellet to pass by the light barriers, the time division of the oscilloscope and the uncertainty regarding the position of the light barriers. This last one is measured with a standard ruler, with a minimum division of $\Delta \mathrm{s}=1 \mathrm{~mm}$.

$\Delta v_{p}=\left|\frac{\partial v_{p}}{\partial s}\right| \times \Delta s+\left|\frac{\partial v_{p}}{\partial t}\right| \times \Delta t=\left|\frac{1}{t}\right| \times \Delta s+\left|-\frac{s}{t^{2}}\right| \times \Delta t$

Where:

$$
s=0.072 \mathrm{~m}
$$


$\Delta s=10^{-3} \mathrm{~m}$

$\Delta t=10^{-6} \mathrm{~s}$

Table III shows the uncertainty in the pellet speed measurement.

TABLE III: Pellet speed uncertainty

\begin{tabular}{llll}
\hline Speed $[\mathrm{m} / \mathrm{s}]$ & Time $[\mathrm{s}]$ & Error $[\mathrm{m} / \mathrm{s}]$ & Relative error $(\%)$ \\
\hline 400 & 0.00018 & 8 & 1.9 \\
450 & 0.00016 & 9 & 2 \\
500 & 0.00014 & 10 & 2.1 \\
550 & 0.00013 & 12 & 2.2 \\
600 & 0.00012 & 13 & 2.2 \\
650 & 0.00011 & 15 & 2.3 \\
700 & 0.00010 & 17 & 2.4 \\
\hline
\end{tabular}

\section{REFERENCES}

${ }^{1}$ H. W. Kugel et al., "The effect of lithium surface coatings on plasma performance in the National Spherical Torus Experiment” Phys. Plasmas 15, 056118, 2008 DOI: 10.1063/1.2906260

${ }^{2}$ J. S. Hu et al., "New Steady-State Quiescent High-Confinement Plasma in an Experimental Advanced Superconducting Tokamak" Phys. Rev. Lett. 114, 055001, 2015, DOI: 10.1103/PhysRevLett.114.055001

${ }^{3}$ T. H. Osborne et al., "Enhanced H-mode pedestals with lithium injection in DIII-D" Nucl. Fusion 55(6), 063018, 2015, DOI: 10.1088/0029-5515/55/6/063018

${ }^{4}$ P.T. Lang, P. Cierpka, R.S. Lang, S.M. Egorov, B.V. Kuteev, P.V. Reznichenko, and V. Yu. Sergeev. "Compact gas gun injection system for variable sized solid pellets" Review of Scientific Instruments, 65(7), 1994.

${ }^{5}$ L. Crocco. "Fundamentals of Gas Dynamics" Princeton University Press, 1958.

${ }^{6}$ M. Willensdorfer, E. Wolfrum, R. Fischer, J. Schweinzer, M. Sertoli, B. Sieglin, G. Veres, F. Aumayr, and the ASDEX Upgrade Team. "Improved chopping of a lithium beam for plasma edge diagnostic at ASDEX upgrade" Review of Scientific Instruments, 83(2), 2012.

${ }^{7}$ E. Wolfrum, F. Aumayr, D. Wutte, HP. Winter, E. Hintz, D. Rusbüldt, and R. P. Schorn. "Fast lithium-beam spectroscopy of tokamak edge plasmas" Review of Scientific Instruments, 64(8):2285-2292, 1993.

${ }^{8}$ S.K. Rathgeber, L. Barrera, G. Birkenmeier, R. Fischer, W. Suttrop, and the ASDEX Upgrade Team. "Investigations on the edge kinetic data in regimes with type-I and mitigated ELMs at ASDEX upgrade" Nuclear Fusion, 54(9):093011, 2014. 